\title{
Different Risk Target Volumes for Nasopharyngeal Carcinoma Treated with Simultaneous Integrated Boost Intensity-Modulated Radiotherapy
}

\author{
Fangzheng Wang ${ }^{1,2,3,4}{ }^{\bowtie}$, Chuner Jiang1,5,6, ${ }^{*}$, Lei Wang ${ }^{1,2,3,4}$, Fengqin Yan 1,2,3,4, Yongfeng Piao, 1,2,3,4, Zhimin \\ $\mathrm{Ye}^{1,2,3,4}$, Min $\mathrm{Xu}^{1,5,6}$, Jiping Liu ${ }^{1,5,6}$, Zhenfu $\mathrm{Fu}^{1,2,3,4}$ and Yangming Jiang7
}

1. Department of Radiation Oncology, Cancer Hospital of the University of Chinese Academy of Sciences, Zhejiang Hangzhou 310022, People's Republic of China.

2. Institute of Cancer and Basic Medicine (ICBM), Chinese Academy of Sciences, Zhejiang Hangzhou 310022, People's Republic of China.

3. Department of Radiation Oncology, Zhejiang Cancer Hospital, Zhejiang Hangzhou 310022, People's Republic of China.

4. Key Laboratory of Radiation Oncology of Zhejiang Province, Zhejiang Hangzhou 310022, People's Republic of China.

5. Department of Breast Tumor Surgery, Cancer Hospital of the University of Chinese Academy of Sciences, Zhejiang Hangzhou 310022, People's Republic of China.

6. Department of Breast Tumor Surgery, Zhejiang Cancer Hospital, Zhejiang Hangzhou 310022, People's Republic of China.

7. Department of Digital Earth, Institute of Remote Sensing and Digital Earth, CAS, Beijing, 100101, People's Republic of China.

*These authors have contributed equally to this work.

$\square$ Corresponding authors: Zhenfu Fu, Tel.: 86-571-88128195; E-mail: fzf1000@163.com; and Fangzheng Wang, Tel.: 86-571-88128192; E-mail: wangfz76@126.com.

( ) The author(s). This is an open access article distributed under the terms of the Creative Commons Attribution License (https://creativecommons.org/licenses/by/4.0/). See http://ivyspring.com/terms for full terms and conditions.

Received: 2020.03.07; Accepted: 2020.06.15; Published: 2020.07.06

\begin{abstract}
Background and Objectives: Although intensity-modulated radiotherapy (IMRT) provides promising survival advantages and fewer late complications in patients with nasopharyngeal cancer (NPC), appropriated target volumes and prescribed doses are still being explored. This study aimed to propose different risk target volumes and corresponding prescribed doses in our center and to evaluate the physical basis and efficacy of this protocol based on the long-term survival of NPC patients.

Methods and Materials: We retrospectively assessed patients with histology-proven non-metastatic NPC treated with definitive IMRT using our protocol of different risk target volumes and corresponding prescribed doses based on the orderly stepwise pattern of tumor spread. We described the delineation for different risk target volumes and the design of IMRT planning for an NPC case. Additionally, we compared the dosimetric distributions between the China protocol and our protocol through two NPC cases. The patterns of failure and locoregional control were the primary endpoints. All survival outcomes were calculated using the Kaplan-Meier method.

Results: From January 2013 to December 2014, a total of 335 patients were treated; the median follow-up for patients who survived was 70 months. All patients completed IMRT using our protocol. Twenty-five patients developed locoregional recurrence, and all recurrences occurred within the high-dose target volumes. The rates of locoregional recurrence-free survival, distant metastasis-free survival, progression-free survival, and overall survival at 5 years were $92.2 \%, 92.1 \%, 85.9 \%$, and $86.3 \%$, respectively. The biological effective doses of the prescribed doses in our protocol were similar to those of the China and 0615 protocols. Moreover, our protocol offered a reduction in D1 and D2 in the primary gross tumor volume (GTV), while V30 and V40 in normal tissues were lower.

Conclusion: Our protocol of different risk target volume delineations and corresponding prescribed doses based on the stepwise pattern of tumor spread resulted in favorable locoregional control with no relapse outside the GTV.
\end{abstract}

Key words: nasopharyngeal carcinoma; IMRT; target volume; dose; long-term survival 


\section{Introduction}

Nasopharyngeal cancer (NPC), a unique malignancy of the head and neck, is endemic to southeast Asia, northern Africa, and middle Europe where the acute incidence is $15-50$ cases per 100,000 annually [1]. The latest GLOBOCAN data [2] in 2018 reported that 129,097 patients were newly diagnosed with NPC worldwide. Of these patients, $47.7 \%$ were in China.

Due to the high sensitivity to radiation and the complicated anatomical structure of the nasopharynx, radiation therapy (RT) is regarded as the mainstay of treatment for NPC. Previously, conventional two-dimensional RT was a common technique for NPC. In early reports, the 5-year survival rate of NPC after two-dimensional RT was $67-76 \%$ [3-7]. However, late complications, including carotid stenosis, optic neuropathy, and brain necrosis, reduced the quality of life in long-term survivors [8-10].

Compared with two-dimensional RT, intensitymodulated radiation therapy (IMRT) could improve the dose covering the clinical target volume (CTV) in three dimensions while protecting the normal tissues around the CTV [11]. ]. Moreover, IMRT provides favorable local control and survival outcomes in NPC [12-18]. In contrast to non-IMRT techniques, IMRT improves cancer-specific survival for head and neck cancer according to the Surveillance, Epidemiology, and End Results (SEER) databases, especially in NPC patients [19]. IMRT can be implemented using a sequential (SEQ) or simultaneous integrated boost (SIB) protocol. SEQ IMRT uses a two-phase shrinkage technique, while SIB IMRT delivers different dose levels to different target volumes. Compared with SEQ IMRT, SIB IMRT yields more satisfactory dosimetric outcomes for nearby critical organs and is regarded as the standard therapy for NPC [20-22]. In addition, whole-field SIB IMRT provides more dosimetric benefits for the larynx and fewer set-up errors than junction IMRT with a conventional RT field [23].

Currently, due to the introduction of Magnetic Resonance (MR) and induction chemotherapy (IC), IMRT has significantly improved the survival rate of NPC to $80-85 \%$ [12-14]. A recent study reported that the locoregional relapse-free survival (LRRFS), distant metastasis-free survival (DMFS), and disease-specific survival (DSS) of 865 NPC patients in 2001-2008 were $92 \%, 83.4 \%$, and $78.6 \%$, respectively, 10 years after IMRT [24]. However, insufficient RT dose is still one of the most important factors affecting the therapeutic effect. In many previous protocols [12-14, 16-18], bone mark and wide margins from the GTV of the nasopharynx and cervical lymph nodes were used to encompass the high-dose area. These experiences came from conventional two-dimensional RT techniques. Although IMRT provides encouraging local control and survival outcomes, normal tissues are also exposed to unnecessarily high doses, and some late complications such as hearing loss, carotid stenosis, optic neuropathy, and brain necrosis occur in long-term survivors [8-10, 25]. Ng and colleagues [26] evaluated the effect of dosimetric inadequacy in target volumes on the local control of NPC; the results indicated that if the volume in the gross tumor volume (GTV) below 66.5 Gy was more than $3 \mathrm{cc}$, the 5 -year rate of local failure-free survival dropped to $54 \%$. Both target volumes and prescribed doses are vital for IMRT in NPC. Recently, a global questionnaire study proposed a CTV design scheme for NPC, i.e., the principle of " $5+5 \mathrm{~mm}$ expansion." Based on the GTV, the high-risk CTV is formed by expanding $5 \mathrm{~mm}$, and then the low-risk CTV is formed by a further expansion of $5 \mathrm{~mm}$ [27]. However, there are no available data regarding the expansion of NPC tumors and no radical dose for the GTV. If the CTV is obtained by uniform expansion from the GTV, excessive normal tissue may be irradiated. Therefore, reduced target volumes have been used to apply IMRT in NPC [15-17]. However, optimal target volumes and doses of IMRT in NPC remain unclear due to a lack of available data on the extent of the invasion of the NPC tumor.

Considering the above situation, we designed a protocol for different risk target volumes and corresponding prescribed doses in SIB IMRT based on the characteristics of the gradual spread of the tumor. The main objectives were to introduce our protocol and investigate the long-term results and adverse events in patients with NPC treated with our protocol.

\section{Materials and Methods}

\section{Patients}

Between January 2013 and December 2014, 355 patients hospitalized at the Department of Radiation Oncology at Zhejiang Cancer Hospital were retrospectively reviewed. Eligible patients met the following criteria: (i) biopsy-proven NPC, (ii) no metastasis occurred, (iii) received SIB IMRT using our protocol, (iv) completion of treatment, and (v) no previous anti-cancer treatment. Patients who did not complete definitive IMRT, those without metastasis at diagnosis, or those who had received previous anti-cancer treatment were excluded. This retrospective study was approved by the Medical Ethics Committee and the Institutional Review Board of Zhejiang Cancer Hospital. All treatment protocols 
in this study were carried out in accordance with the National Comprehensive Cancer Network guidelines. Due to the retrospective design of the study, the committee confirmed that informed consent was not required.

\section{Pretreatment evaluations}

Pretreatment evaluations included the following: detailed medical histories, evaluation of performance status, and careful physical examinations. Magnetic resonance imaging (MRI) of the nasopharynx and nasopharyngoscopies was performed. Chest computed tomography (CT), bone scans, abdominal ultrasound, and hematology and biochemistry tests were conducted 1 week before treatment. Positron emission tomography scans and abdominal CT scans were performed as clinically indicated. The 2010 American Joint Committee on Cancer staging system and World Health Organization classification were recommended for use in this study.

\section{SIB IMRT}

\section{Body position fixation and scanning requirements}

All patients receiving SIB IMRT were immobilized in the supine position using thermoplastic masks. CT scans with intravenous contrast using 2.5-mm slices from the head to a level 2 $\mathrm{cm}$ below the sternoclavicular joints were performed for planning. Intravenous contrast was administered at approximately $1-2 \mathrm{~mL} / \mathrm{kg} / \mathrm{s}$.

\section{Names and relationship of different risk target volumes}

Based on the orderly stepwise pattern of tumor spread, we designed three or four risk target volumes in the pathway of NPC tumor invasion including the peripheral (Figure 1A), upper, and lower structures (Figure 1B). The corresponding target volumes included high-risk planning target volume of nasopharyneal GTV (PGTVnx) or planning target volume of GTV in cervical lymph nodes (PGTVnd) encompassing GTV of nasopharynx (GTVnx) or GTV of cervical lymph nodes (GTVnd), intermediate-risk planning target volume of nasopharyx (PTVnx) or planning target volume of neck area (PTVna) encompassing CTV of nasopharynx (CTVnx) or CTV of neck area (CTVna), and low-risk planning target volume (PTV) encompassing CTV. If the retropharyngeal lymph nodes (RLNs) were large or resistance to IC, we add a higher risk target volume for PTV of GTV in retropharyngeal lymph nodes (PGTVrpn) encompassing GTV of retropharyngeal lymph nodes (GTVrpn). The relationship of the three to four risk tumor volumes such as PGTVnx (PGTVrpn), PGTVnd, PTVnx, PTVna, and PTV is shown in Figure 1.

\section{Definitions of the different risk target volumes}

According to the orderly stepwise pattern of tumor spread, we designed the different risk target volumes as follows. GTV referred to the macroscopic tumor extent found in clinical and imaging baseline examinations or before treatment. The primary tumor extent including the metastatic retropharyngeal lymph nodes (RLNs) was named the GTVnx, and the metastatic lymph nodes of the neck were named the GTVnd. If the RLNs were large and resistant to IC, we add a GTVrpn into the GTVnx. Based on the above for each GTV, we automatically expanded the margin by an additional 1-3 $\mathrm{mm}$ in three dimensions to get the corresponding PTVs, including PGTVnx, PGTVnd, and PGTVrpn.

B

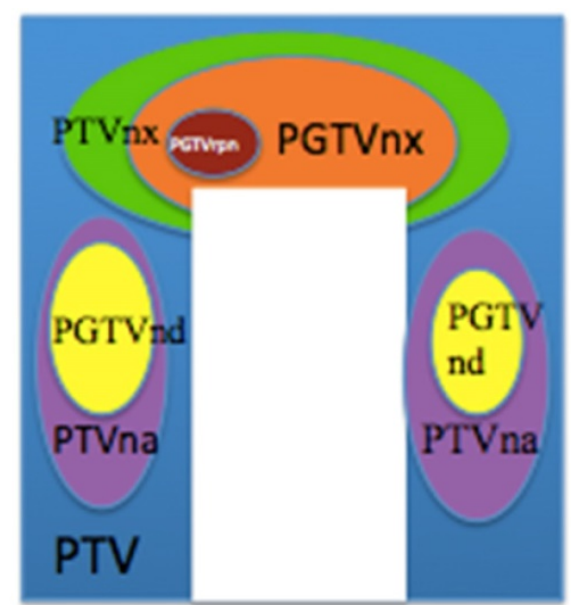

Figure 1. The different risk target volumes. (A) Cross section; (B) coronary position. Red: PGTVrpn; orange: PGTVnx; yellow: PGTVnd; green: PTVnx; pink: PTVna; blue: PTV. Abbreviations: PGTVrpn: planning target volume of GTV in retropharyngeal lymph nodes; PGTVnx: planning target volume of nasopharyngeal GTV; PGTVnd: planning target volume of GTV in cervical lymph nodes; PTVnx: planning target volume of nasopharynx; PTVna:planning target volune of neck area; PTV: planning target volume. 


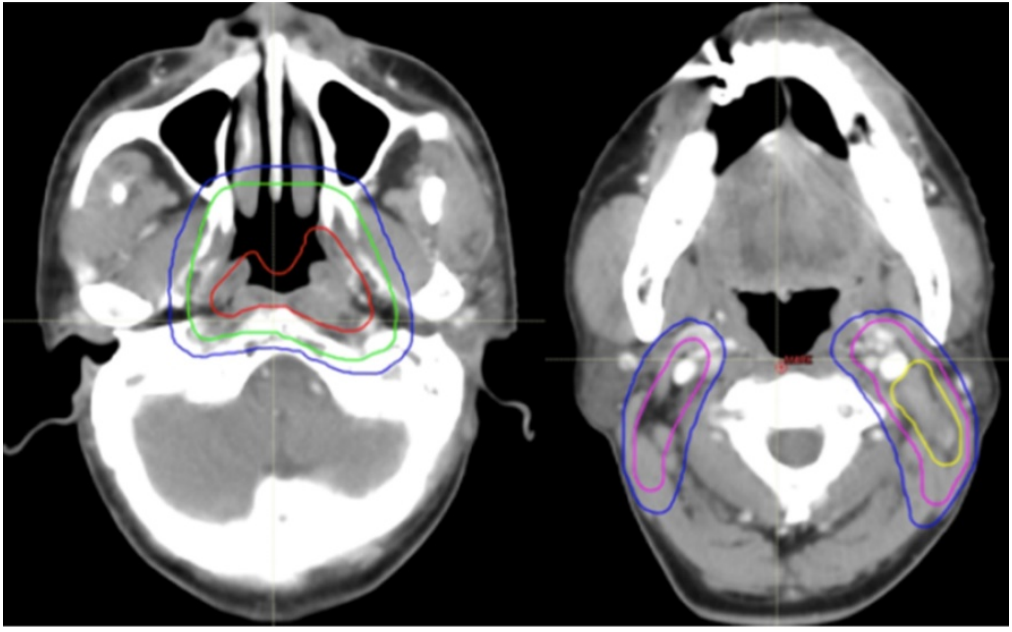

Figure 2. Selected computed tomography slices to demonstrate the delineation of different risk target volumes. Red: PGTVnx; yellow: PGTVnd; green: PTVnx; pink: PTVna; blue: PTV1. Abbreviations: PGTVnx: planning target volume of nasopharyngeal GTV; PGTVnd: planning target volume of GTV in cervical lymph nodes; PTVnx: planning target volume of nasopharynx; PTVna: planning target volune of neck area; PTV: planning target volume 1.

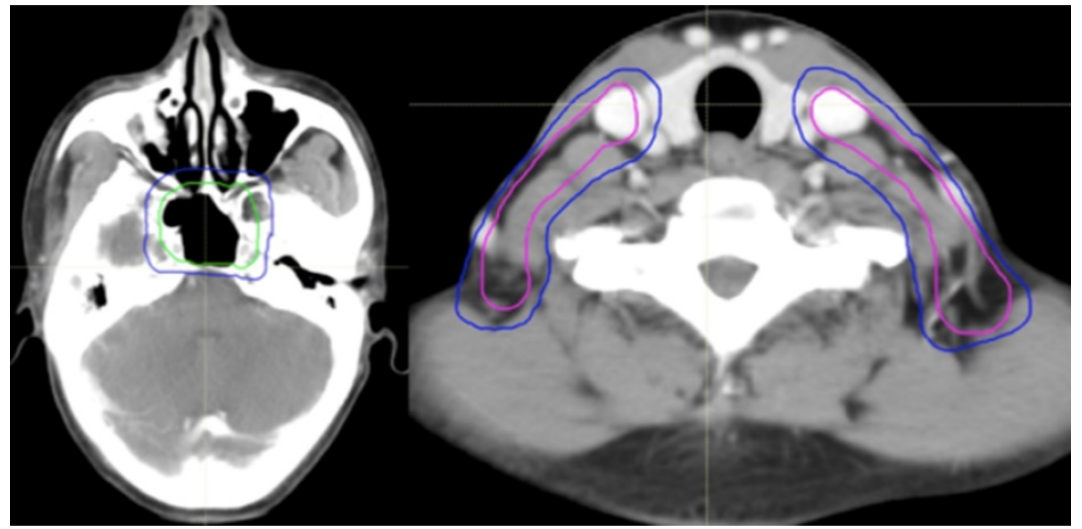

Figure 3. Selected computed tomography slices to demonstrate the delineation of different risk target volumes. Red: PGTVnx; yellow: PGTVnd; green: PTVnx; pink: PTVna; blue: PTV1. Abbreviations: PGTVnx: planning target volume of nasopharyngeal GTV; PGTVnd: planning target volume of GTV in cervical lymph nodes; PTVnx: planning target volume of nasopharynx; PTVna: planning target volune of neck area; PTV: planning target volume 1.

The CTV included the high-risk CTV (CTVnx and CTVna) and low-risk CTV according to the orderly stepwise pattern of tumor spread and the risk region potentially involved around the nasopharyngeal cavity. For stage T1-2, CTVnx was defined as GTVnx plus a 5-7-mm margin encompassing the entire nasopharyngeal mucosa plus $5 \mathrm{~mm}$ of the submucosal volume. For stage T3-4, CTVnx was defined as GTVnx plus a 5-7-mm margin encompassing the entire nasopharyngeal mucosa plus $5 \mathrm{~mm}$ of the submucosal volume, parapharyngeal space, pterygoid fossae, and foramen lacerum. PTVnx was created automatically by adding a 1-3-mm margin in three dimensions. For stage N0, CTVna was defined as lymphatic drainage clearance in level II of the bilateral neck, while for stage N1-3, CTVna included GTVnd plus a 2-5-mm margin, the ipsilateral lymph drainage space $2 \mathrm{~cm}$ below the GTVnd, and the contralateral lymphatic drainage clearance in level II. PTVna was created automatically by adding a 1-3-mm margin. The low-risk CTV included the CTVnx plus a 5-10 mm margin, CTVnx plus a 2-5-mm margin, and the prophylactic low-risk neck irradiation area. The low-risk PTV was defined as the low-risk CTV plus a 3-mm margin.

All of the PTVs, including PGTVnx, PGTVnd, PTVnx, PTVna, and PTV, were trimmed so as not to be delineated outside of the skin surface. Table 1 and Figures 2-4 demonstrate the delineations of different risk target volumes using our protocol.

\section{General principles of different risk target volume design}

In one CT image slice, if there is a GTV, the peripheral structures of the NPC tumor (primary tumor and cervical lymph nodes) invasion should be divided into three tumor volumes of high-, medium-, and low risk such as the PGTVnx/nd, PTVnx/na, and PTV. Moreover, three prescribed dose levels are given for the corresponding risk target volumes.

In one CT image slice, if there is a high-risk area but no GTV, two risk target areas and two prescription dose levels for medium- and low risk should be designed. 
In one CT image slice, if there is neither a GTV nor a high-risk area but it includes a low-risk area, a target area and a prescription dose for low risk should be designed.

\section{Dose prescription}

Doses of 70.5, 70.5, 63, 60, and 51 Gy in 30 fractions were administered to the PGTVnx, PGTVnd, PTVnx, PTVna, and PTV, respectively. If PGTVrpn was added into PGTVnx, a dose of 73.5 Gy in 30 fractions was delivered. SIB IMRT was performed once daily, in five fractions per week, over 6 weeks according to the IMRT planning.

\section{Delineation of organs at risk}

Critical normal structures, including the brainstem, spinal cord, parotid glands, optic nerves, chiasm, lens, eyeballs, temporal lobes, temporomandibular joints, mandible, and hypophysis, were contoured and set as organs at risk (OARs) during optimization.

\section{Chemotherapy}

Out of 335 patients, 302 received three-weekly platinum-based IC, 313 underwent three-weekly concurrent chemotherapy with cisplatin, and 199 received adjuvant chemotherapy (AC). The available IC regimens included TPF (docetaxel $60 \mathrm{mg} / \mathrm{m}^{2} /$ day on day 1 , cisplatin $25 \mathrm{mg} / \mathrm{m}^{2} /$ day on days $1-3$, and 5 -fluorouracil $500 \mathrm{mg} / \mathrm{m}^{2} /$ day on days 1-3), TP (docetaxel $60 \mathrm{mg} / \mathrm{m}^{2} /$ day on day 1 , cisplatin 25 $\mathrm{mg} / \mathrm{m}^{2} /$ day on days $1-3$ ), GP (gemcitabine 1,000 $\mathrm{mg} / \mathrm{m}^{2} /$ day on days 1 and 8 , cisplatin $25 \mathrm{mg} / \mathrm{m}^{2} /$ day on days 1-3), and FP (cisplatin $25 \mathrm{mg} / \mathrm{m}^{2} /$ day on days $1-3$, 5 -fluorouracil $500 \mathrm{mg} / \mathrm{m}^{2} /$ day on days $1-3$ ). The one to two cycles of AC consisted of cisplatin and 5-fluorouracil.

\section{Plan evaluation}

Plans were compared by target coverage according to the cross-section dose distribution and the dose-volume histogram (DVH) of the targets. Parameters of the DVH were evaluated as follows: 1) doses received by $95 \%$ and $90 \%$ of the volumes of the PTV (D95 and D90, respectively), maximum PTV dose (Dmax), minimum PTV dose (Dmin), and mean PTV dose; 2) maximum OARs dose and the volume of OARs receiving a high dose; and 3) the volume received 30 or 40 Gy of the normal tissues.

\section{Adverse events and survival evaluation}

Adverse events were assessed according to the common toxicity criteria of the National Cancer Institute. Survival was calculated from the date of diagnosis to the date of the most recent follow-up, recurrence, or death. The pattern of failure was defined according to the first site of failure: recurrence of the primary tumor or metastasis to regional lymph nodes was regarded as locoregional failure, and metastasis to any site beyond the primary tumor and regional lymph nodes was defined as distant failure.

Table 1. Delineation of different risk target volumes using our protocol

\begin{tabular}{|c|c|}
\hline Target volume & Definition \\
\hline GTV in NP & GTVnx \\
\hline GTV of LN & GTVnd \\
\hline High-risk target volumes & $\begin{array}{l}\text { PGTVnx }=\text { GTVnx }+0-3 \mathrm{~mm}+\text { whole NP }(5 \mathrm{~mm} \text { submucosal }) \\
\text { PGTVnd }=\text { GTVnd }+3 \mathrm{~mm}\end{array}$ \\
\hline $\begin{array}{l}\text { Intermediate-risk target volume } \\
\text { for primary tumor }\end{array}$ & $\begin{array}{l}\text { T1-2: CTVnx }=\text { GTVnx }+5-7 \mathrm{~mm} \\
\text { T3-4: CTVnx }=\text { GTVnx }+5-7 \mathrm{~mm}+\text { whole NP }(5 \mathrm{~mm} \text { submucosal })+\text { pterygoid + foramen lacerum } \\
\text { PTVnx }=\text { CTVnx }+1-3 \mathrm{~mm}\end{array}$ \\
\hline Nasal cavity-posterior part & $3 \mathrm{~mm}$ from the choana \\
\hline Maxillary sinuses-posterior part & $3 \mathrm{~mm}$ from the posterior wall \\
\hline Posterior ethmoid sinus & If invasion, \\
\hline Skull base & Cover foramina ovale, rotundum, and lacerum \\
\hline Cavernous sinus & Cover side involved only if T3-4 \\
\hline Pterygoid fossae & + \\
\hline Parapharyngeal spaces & Full coverage \\
\hline Sphenoid sinus & Inferior half if no invasion; whole if invasion \\
\hline Clivus & Anterior third if no invasion; whole if invasion \\
\hline $\begin{array}{l}\text { Intermediate-risk target volume } \\
\text { for cervical lymph node }\end{array}$ & $\begin{array}{l}\text { CTVna: cover bilateral level II plus VA if N0; cover bilateral level II, VA, plus at least one level ipsilateral below the involved levels } \\
\text { PTVna = CTVna }+1-3 \mathrm{~mm}\end{array}$ \\
\hline Bilateral RP, level II, III, IVa & Bilateral level II plus VA if N0; bilateral level II, VA, plus at least one level ipsilateral below the involved levels \\
\hline Level IB & $\begin{array}{l}\text { IB } L N+v e \\
\text { IIA } L N+\text { ve } \\
\text { Invaded structure that drains to level IB as first echelon site }\end{array}$ \\
\hline Low-risk target volume & $\begin{array}{l}\text { CTV: CTV nx }+3-7 \mathrm{~mm} \text { for primary site; CTVna }+2-5 \mathrm{~mm} \text { for cervical LN; cover lower neck and supraclavicular if } \mathrm{N} 1-3 \text { or omit if } \mathrm{N} 0 \\
\text { PTV = CTV }+1-3 \mathrm{~mm}\end{array}$ \\
\hline
\end{tabular}

GTV: gross tumor volume; NP: nasopharynx; LN: lymph node; CTV: clinical target volume; PTV: planning target volume; GTVnx: GTV of nasopharynx; GTVnd: GTV of cervical lymph nodes; PGTVnx: planning target volume of nasopharyngeal GTV; PGTVnd: planning target volume of GTV in cervical lymph nodes; CTVnx: CTV of nasopharynx; PTVnx: planning target volume of CTV; CTVna: clinical target volume of neck area. 


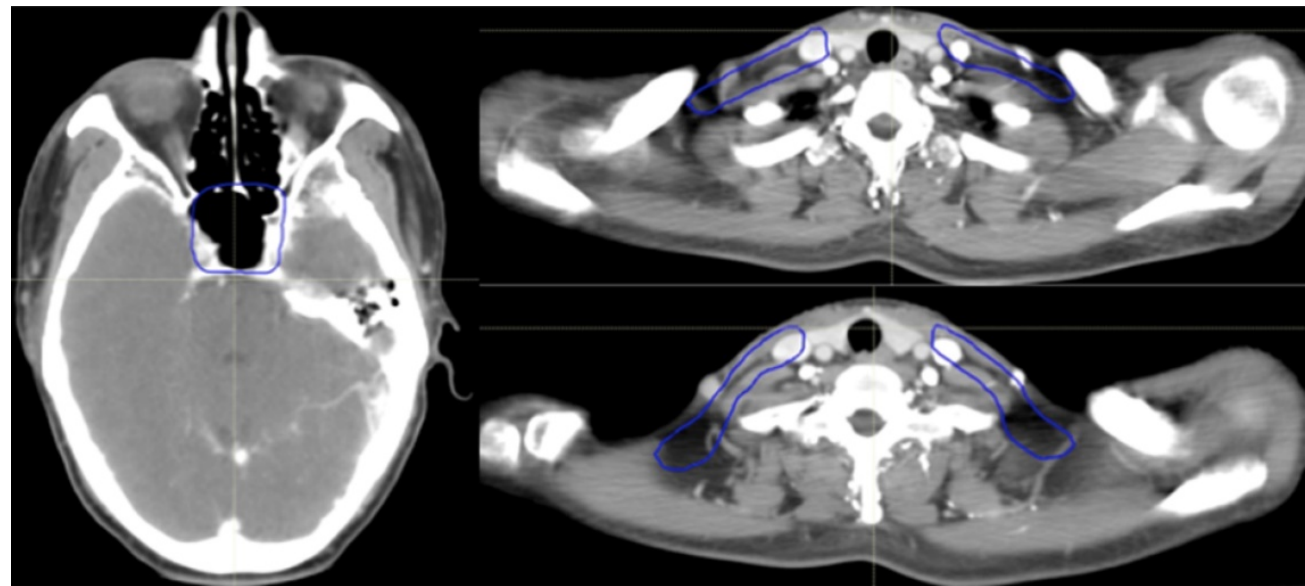

Figure 4. Selected computed tomography slices to demonstrate the delineation of low-risk target volumes. Red: PGTVnx; yellow: PGTVnd; green: PTVnx; pink: PTVna; blue: PTV1. Abbreviations: PGTVnx: planning target volume of nasopharyngeal GTV; PGTVnd: planning target volume of GTV in cervical lymph nodes; PTVnx: planning target volume of nasopharynx; PTVna: planning target volume of neck area; PTV: planning target volume 1.

Table 2. Baseline characteristics of the 335 newly diagnosed NPC patients enrolled in the study

\begin{tabular}{lll}
\hline Characteristic & Number of patients & $\%$ \\
\hline Age at diagnosis & & \\
Range (years) & $17-79$ & \\
Median age (years) & 50 & \\
Sex & & \\
Male & 240 & 71.6 \\
Female & 95 & 28.4 \\
T stage & \\
T1 & & \\
T2 & 13 & 3.9 \\
T3 & 101 & 30.1 \\
T4 & 143 & 42.7 \\
N stage & \\
N0 & 78 & 23.3 \\
N1 & & \\
N2 & 4 & 1.2 \\
N3 & 64 & 19.1 \\
Clinical stage* & 235 & 70.1 \\
II & 32 & 9.6 \\
III & 26 & \\
IV & 208 & 7.8 \\
IC regimens & 101 & 62.1 \\
TPF & & 30.1 \\
TP & 54 & \\
GP & 155 & 16.1 \\
PF & 71 & 46.3 \\
No & 22 & 21.2 \\
Treatment modality & 33 & 6.6 \\
IC+CRT+AC & 165 & 9.8 \\
IC+IMRT+AC & 14 & \\
IC+CRT & 115 & 49.3 \\
IC+IMRT & 8 & 4.2 \\
CRT & 13 & 34.3 \\
CRT+AC & 20 & 3.9 \\
\hline & & 5.9 \\
\hline
\end{tabular}

IC: induction chemotherapy; CRT: concurrent chemoradiotherapy; AC: adjuvant chemotherapy; IMRT: intensity-modulated radiotherapy; TPF: docetaxel/ cisplatin/fluorouracil; TP: docetaxel/cisplatin; GP: gemcitabine/fluorouracil; FP: cisplatin/fluorouracil.

*American Joint Committee on Cancer/International Union against Cancer staging system, seventh edition.

\section{Endpoints and statistical analysis}

The endpoints of the present study were LRRFS (time from the date of confirmed NPC to locoregional failure), DMFS (time from the date of confirmed NPC to distant metastasis), progression-free survival (PFS) (time from the date of confirmed NPC to progression), OS (time from the date of confirmed NPC to the last follow-up), and acute adverse events from IC and IMRT. If patients relapsed or developed metastasis, they underwent salvage therapy as determined by their physicians. The Kaplan-Meier method was used to generate LRRFS, DMFS, PFS, and OS curves. All data were analyzed using SPSS Statistics version 22.0 (IBM Corp, Armonk, NY).

\section{Results}

\section{Patient characteristics}

From May 2008 to April 2014, the clinical data of 332 untreated, newly diagnosed NPC patients who were initially treated with additional IC followed by SIB IMRT in the Department of Radiation Oncology, Zhejiang Cancer Hospital (Hangzhou, People's Republic of China) were collected and retrospectively reviewed. The basic characteristics of the patients are summarized in Table 2. All patients completed a full course of radical IMRT and received one to four cycles of IC.

\section{Delineation of different risk target volumes and IMRT planning}

To illustrate our protocol of different risk target volumes, a histology-proven NPC patient was used to describe the different risk target volumes and the corresponding doses in IMRT planning. The MRI of this patient indicated that the primary tumor had invaded the parapharyngeal space, bilateral metastatic RLNs had occurred, and multiple metastatic lymph nodes were observed in the bilateral cervical and supraclavicular regions (Figure 5A). 
Therefore, the stage was T2N3M0. The patient received three cycles of TP-based IC. After CT simulation following the third IC, we delineated three risk target volumes including high-risk PGTVnx and GTVnd, intermediate-risk PTVnx and PTVna, and low-risk PTV. The IMRT planning was designed by an experienced physicist. Dose distribution was even (Figure 5B). The DVH showed that the prescribed doses met our requirements (Figure 5C).

\section{Patterns of failure}

Among all patients, 47 experienced treatment failure. Twenty-one had locoregional recurrence within the high-dose GTV. Twelve patients developed regional relapse only, and 10 received salvage surgery; eight patients experienced local recurrence only, and one had both local and regional relapse. Twenty-two patients experienced $\geq 1$ distant metastasis, 21 of whom died from disease progression. Four patients experienced locoregional relapse and distant metastasis. Regarding the metastatic location, six patients developed pulmonary metastasis only, eight experienced bone metastases, two developed hepatic metastasis and six developed multiple organ metastases.
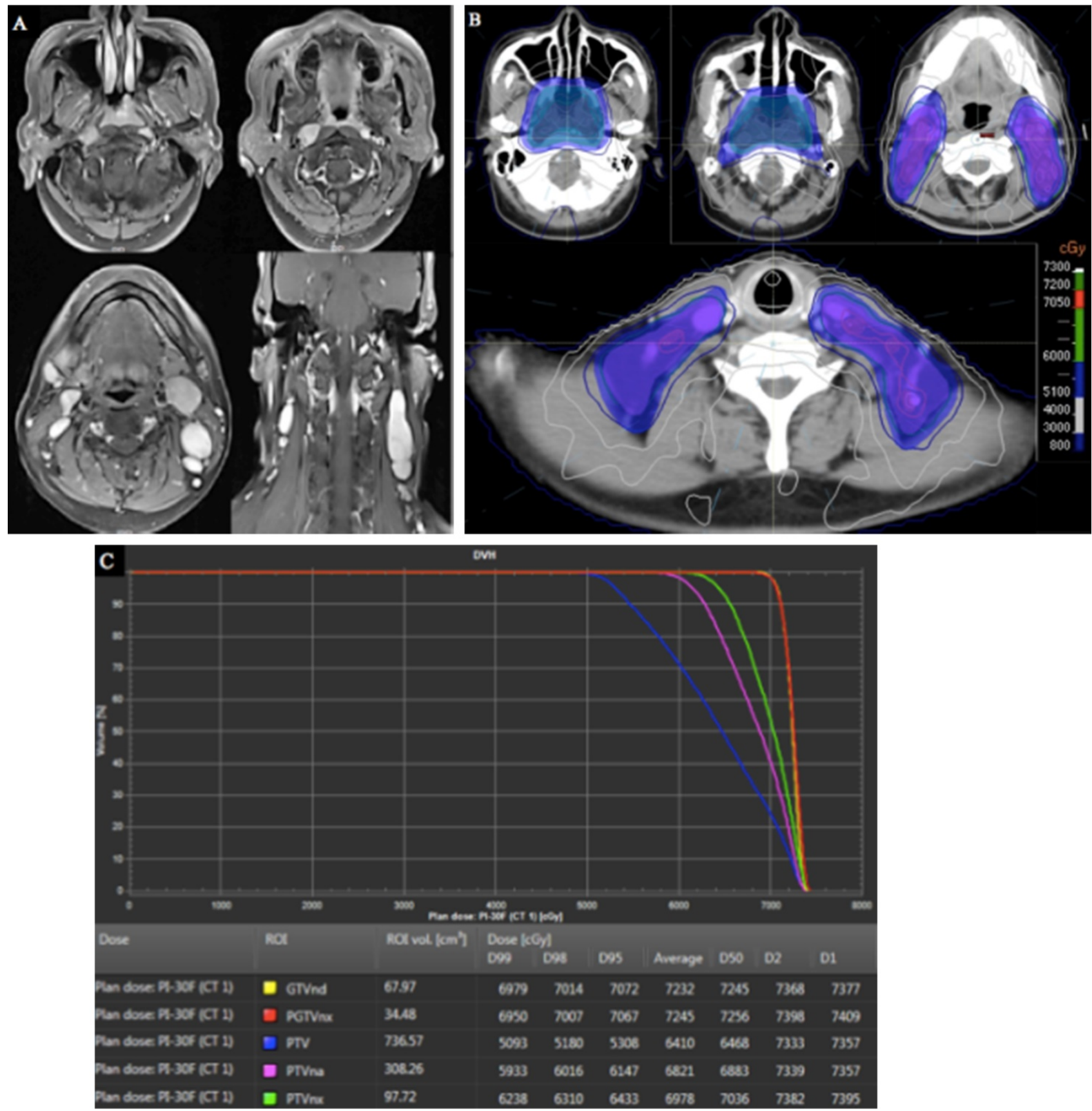

Figure 5. Target volumes and IMRT planning in a patient with stage T2N3MO NPC. (A) MRI of the patient; (B) different risk target volumes and dose distributions; (C) DVH of IMRT. Abbreviations: IMRT: intensity-modulated radiotherapy; NPC: nasopharyngeal cancer; MRI, magnetic resonance imaging; DVH: dose-volume histogram. 

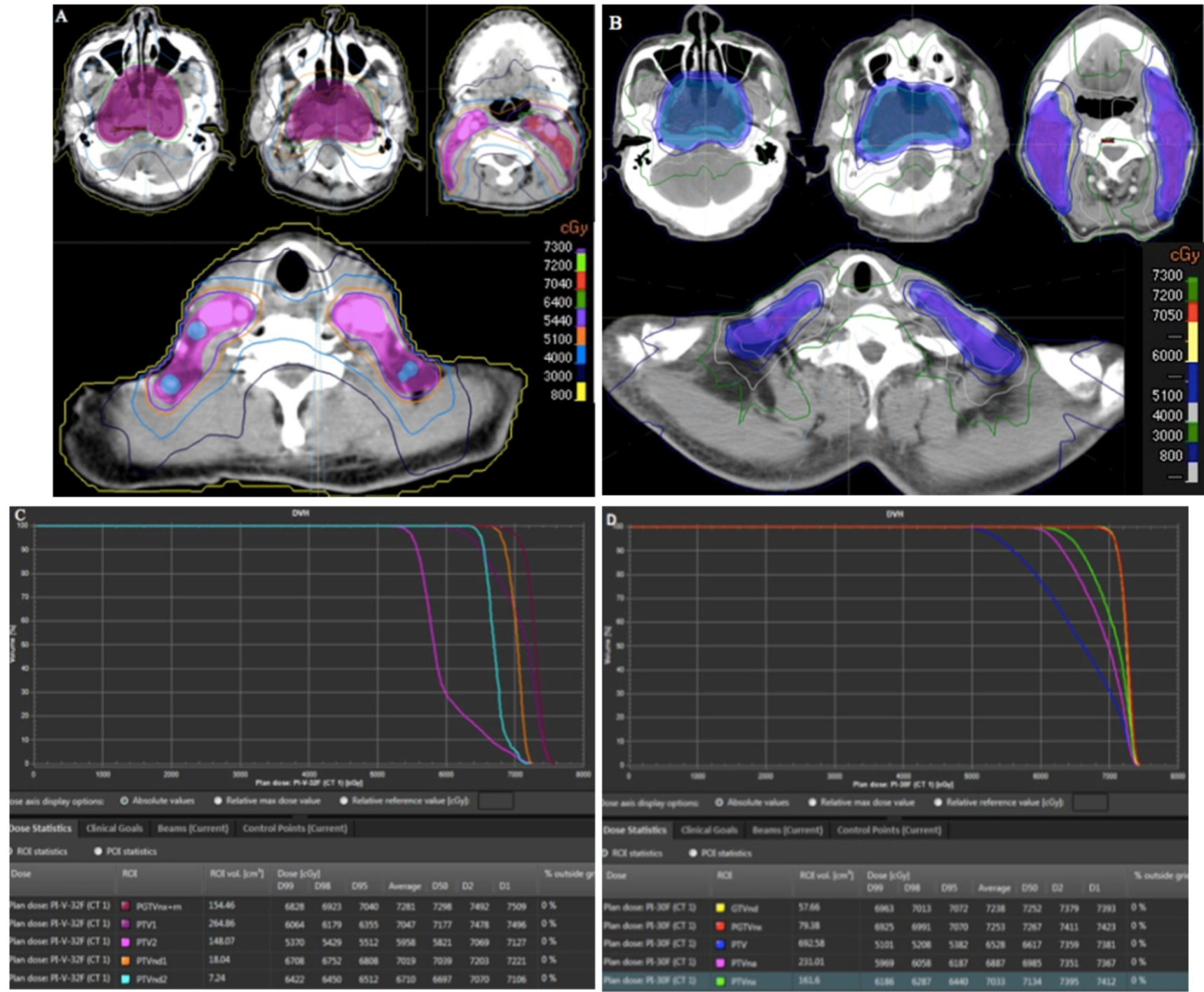

Figure 6. Comparison of target volumes and dose distributions between two protocols. (A) target volumes and dose distributions of the China protocol; (B) target volumes and dose distributions of our protocol; (C) DVH of the China protocol; (D) DVH of our protocol. Abbreviation: DVH: dose-volume histogram.

Table 3. Profile of IC- and RT-related acute toxicities

\begin{tabular}{llllllllll}
\hline Adverse events & \multicolumn{3}{l}{ During IC (n) } & \multicolumn{7}{c}{ During IMRT (n) } \\
\hline Hematologic & 1 & 2 & 3 & 4 & 1 & 2 & 3 & 4 \\
Leukopenia & 35 & 92 & 58 & 36 & 76 & 53 & 32 & 3 \\
Neutropenia & 41 & 62 & 57 & 52 & 68 & 47 & 29 & 5 \\
Anemia & 93 & 29 & 11 & 1 & 42 & 17 & 5 & 0 \\
Thrombocytopenia & 55 & 18 & 8 & 5 & 33 & 13 & 5 & 2 \\
Liver function & 36 & 15 & 6 & 0 & 10 & 5 & 0 & 0 \\
Renal function & 9 & 2 & 1 & 0 & 0 & 0 & 0 & 0 \\
Non-hematologic & & & & & & & & \\
Mucositis & 25 & 16 & 6 & 1 & 181 & 123 & 23 & 0 \\
Dermatitis & 37 & 5 & 0 & 0 & 261 & 57 & 11 & 0 \\
Diarrhea & 31 & 19 & 7 & 0 & 13 & 2 & 0 & 0 \\
Nausea/vomiting & 82 & 58 & 23 & 2 & 35 & 22 & 13 & 0
\end{tabular}

IC: induction chemotherapy; RT: radiotherapy; IMRT: intensity-modulated radiotherapy.

\section{Long-term survival}

A total of 407 patients were treated with SIB
IMRT. Among them, 36 were excluded from further analysis due to loss to follow-up; thus, 335 participants remained. During the follow-up duration of 70 months, the rates of 3-, 5-, and 7-year LRRFS were $94.1 \%, 92.3 \%$, and 91.3\%, respectively (Figure 7A). The distant metastases-free survival rates at 3-, $5-$, and 7-year were 92.7\%, 92.1\%, and 92.1\%, respectively (Figure 7B). The 3-, 5-, and 7-year progression-free survival rates were $88.2 \%, 85.9 \%$, and $85.0 \%$, respectively (Figure 7C). The rates of OS at $3-, 5-$, and 7-year were 90.1\%, 86.3\%, 82.3\%, respectively (Figure 7D). Furthermore, there were no significant differences in LRRFS between T-stage (Figure 8A) and N-stage (Figure 8B) NPC patients.

\section{Adverse events}

For patients treated with IC or AC, adverse events were recorded as IC- or AC-related acute toxicities, while for patients who received RT with or 
without concurrent chemotherapy (CC), adverse events were recorded as RT-related acute toxicities. If adverse events occurred after a follow-up duration of 6 months, they were recorded as late toxicities. No patient in this study died from treatment-related adverse events.

Table 3 lists the profiles of IC- and RT-related complications. During the period of IC, 94 (28.1\%) and $109(32.5 \%)$ patients experienced grade 3-4 leukopenia and neutropenia, 12 and 13 patients
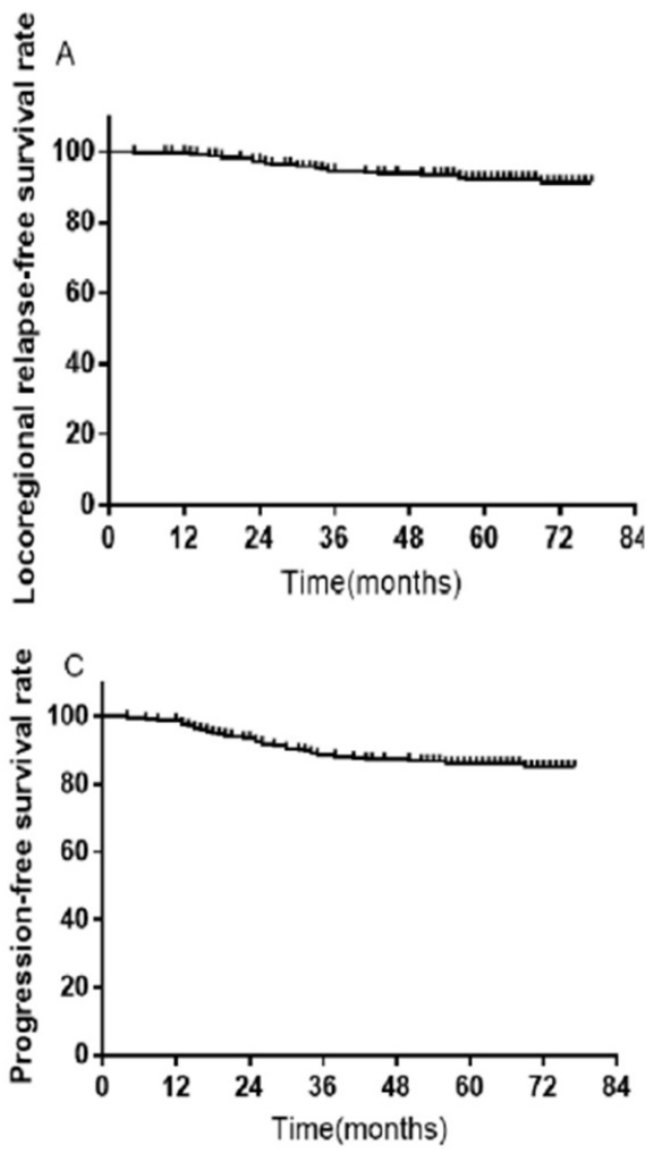

Figure 7. Kaplan-Meier survival curves in 335 NPC patients. (A) LRRFS; (B) DMFS; (C) PFS; (D) OS. Abbreviations: NPC: nasopharyngeal cancer; LRRFS: locoregional relapse-free survival; DMFS: distant metastasis-free survival; PFS: progression-free survival; OS: overall survival.
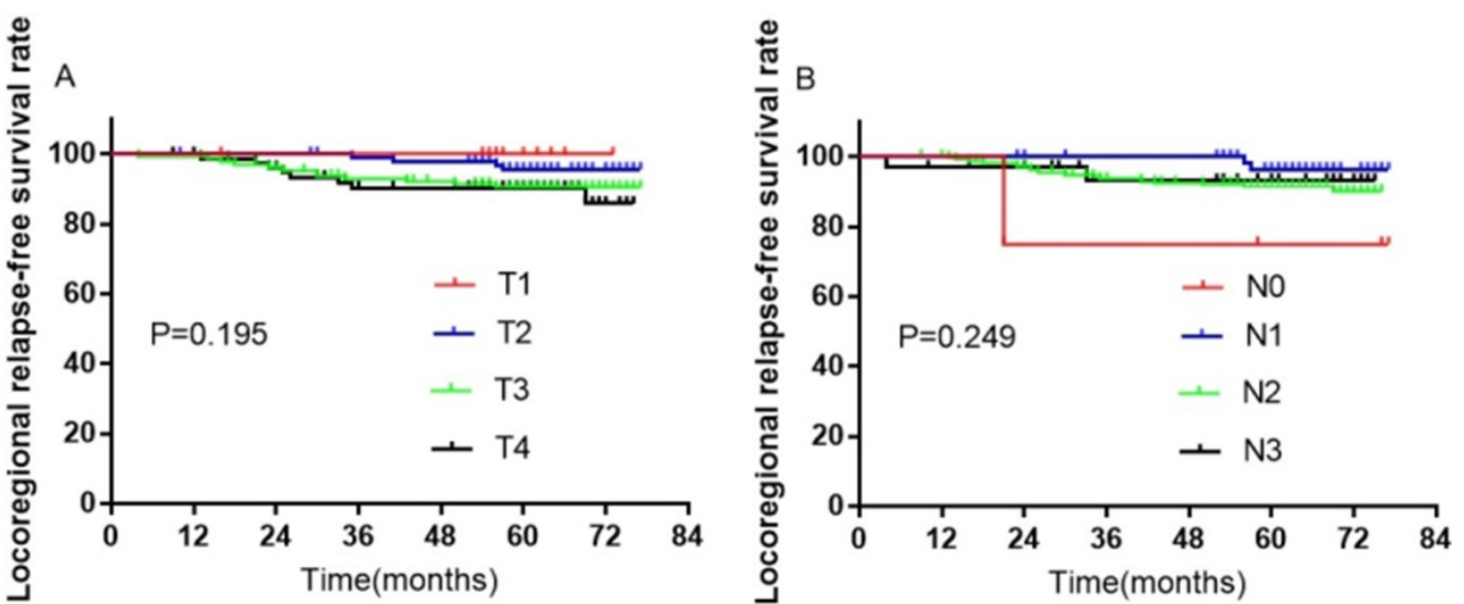

Figure 8. Kaplan-Meier curves of LRRFS in 335 NPC patients with T- or N-stage. (A) T-stage; (B) N-stage. Abbreviations: LRRFS: locoregional relapse-free survival; NPC: nasopharyngeal cancer.

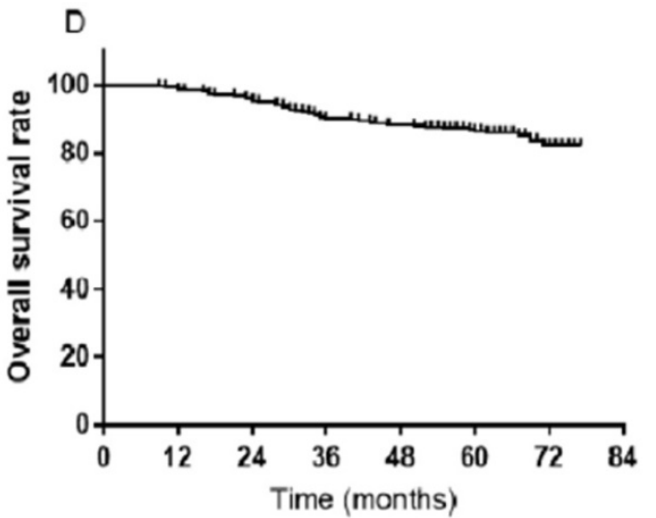

developed grade 3-4 anemia and thrombocytopenia, while the rates of grade 3-4 mucositis, diarrhea, and nausea were $2.1 \%, 2.1 \%$, and $7.5 \%$, respectively. During the period of IMRT, the incidences of grade 34 leukopenia, neutropenia, anemia, and thrombocytopenia were $10.4 \%, 10.1 \%, 1.5 \%$, and $2.1 \%$, respectively. Moreover, 23 and 11 patients experienced grade 3 mucositis and dermatitis, respectively.

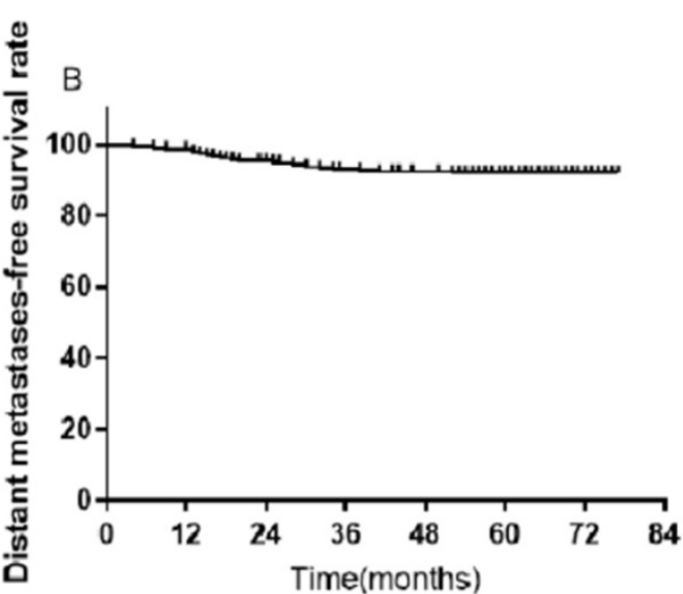


Complications such as dry mouth, hearing loss, carotid stenosis, cranial nerve paralysis, and brain necrosis were regarded as common late adverse events. Of these late adverse events, xerostomia was the most common late complication, and most survivors at the last follow-up experienced mild-to-moderate xerostomia. No grade 2 or higher xerostomia occurred in survivors. However, grade 3 unilateral or bilateral hearing loss was reported in 43 patients $(12.8 \%)$. Five patients developed cranial nerve damage in the posterior group. Carotid stenosis was observed in two patients. Based on MRI, radiation encephalopathy occurred in 16 patients, two of which developed temporal lobe necrosis.

\section{Biological effective dose}

In our protocol, the high-risk PGTVnx and PGTVnd received doses of 70.5-72 Gy and 69-70.5 Gy, respectively; the intermediate-risk PTVnx and PTVna received doses of 63-66 Gy and 60-63 Gy, respectively; and the low-risk PTV received 51-54 Gy. All doses were administrated in 30 fractions. The biological effective doses (BEDs) of our protocol were calculated using the linear-quadratic model according to the early $(\alpha / \beta=10)$ and late $(\alpha / \beta=3)$ tissue response parameters. The BEDs of our protocol were similar to the IMRT dose regimens in the China protocol [28] and 0615 protocol [29] (Table 4). Furthermore, after the equivalent formula conversion, the corresponding equivalent doses in $2 \mathrm{~Gy} /$ fraction (EQDs2) of our protocol were 73.44, 63.53, 60, and $49.73 \mathrm{~Gy}$ in $70.5 \mathrm{~Gy} / 30 \mathrm{~F}, 63 \mathrm{~Gy} / 30 \mathrm{~F}, 60 \mathrm{~Gy} / 30 \mathrm{~F}$ and $51 \mathrm{~Gy} / 30 \mathrm{~F}$, respectively.

Table 4. Comparison of biological equivalent doses among three protocols

\begin{tabular}{lllllll}
\hline \multicolumn{2}{l}{ Variable Present protocol } & \multicolumn{2}{l}{ China protocol } & \multicolumn{2}{l}{$0615 / 0225$ protocol } \\
\hline $\mathrm{a} / \beta$ & Dose & BED & Dose & BED & Dose & BED \\
3 & 70.5 & 125.73 & 70.4 & 122.03 & 70 Gy/33F 119.40 \\
10 & Gy/30F & 88.13 & Gy/32F & 85.89 & & 84.79 \\
3 & 63 Gy/30F 107.1 & 64 Gy/32F & 106.67 & 62.7 & 102.41 \\
10 & & 76.23 & & 85.89 & Gy/33F & 74.61 \\
3 & 60 Gy/30F 100 & 60.8 & 99.31 & 59.4 & 95.04 \\
10 & & 72 & Gy/32F & 72.35 & Gy/33F & 70.1 \\
3 & 51 Gy/30F 79.9 & 54.4 & 85.23 & 50.4 & 80.64 \\
10 & & 59.67 & Gy/32F & 63.65 & Gy/33F & 59.47 \\
\hline
\end{tabular}

\section{Plan comparison}

To evaluate the dosimetric benefits of our protocol, we selected two patients with T4-stage NPC. Target volumes for one patient were delineated using the China protocol, and our protocol was used for the other patient. In the China protocol, doses of 70.4, 70.4, 64, 60.8, and 54.4 Gy in 32 fractions were administered to PGTVnx+rn, PGTVnd1, PGTVns2, PTV1, and PTV2, respectively, while doses of 70.5,
70.5, 63, 60, and 51 Gy in 30 fractions were administered to PGTVnx, PGTVnd, PTVnx, PTVna, and PTV. The two IMRT plans met the requirements. Compared with the China protocol, our strategy led to reductions in the V40 and V30 (Figure 5A and 5B) and D2 and D1 (Figure 6C and Figure 6D). For the three risk target volumes, the high dose was more concentrated in the GTV, and the area receiving a low dose was decreased.

\section{Discussion}

According to the 2019 National Comprehensive Cancer Network guidelines, RT plus concurrent chemotherapy with IC or AC is the preferred treatment for NPC. IMRT has shown remarkable promise in the treatment of NPC. However, optimal target volumes and prescribed doses in IMRT for NPC were unclear. A better understanding of the pathway of NPC tumor spread was used to delineate target tumors and increase survival. Here, we described our protocol of different risk target volumes based on the orderly stepwise pattern of tumor spread. Moreover, we report the long-term survival and adverse events of our protocol. The BEDs of the prescribed doses in our protocol were similar to those of the China and 0615 protocols. Further, our protocol resulted in decreases in D1 and D2 in the GTV, while V30 and $\mathrm{V} 40$ in normal tissues were lower. In addition, our protocol offered acceptable adverse events and favorable long-term survival outcomes with 5-year LRRFS of 92.2\%, 5-year DMFS of 92.1\%, 5-year PFS of $85.9 \%$, and 5 -year OS of $86.3 \%$. Most importantly, no recurrence occurred in the margins or outside of the high-dose area for all survivors with our protocol.

Because of its highly infiltrative nature, NPC tumors easily invade areas of loose tissue and spread along the lacunae and neural foramen. The extent of invasion and the route of spreading have been described based on MRI findings [30]. According to the risk ratios of the invaded anatomic structures around the nasopharynx, Liang et al. [31] and Li et al. [32] divided these anatomic sites into high- $(\geq 50 \%)$, medium- $(5-30 \%)$, and low-risk $(<5 \%)$ regions. Based on the above results, many Chinese experts with extensive experience in the treatment of NPC proposed a protocol in 2010 [28]. This protocol is used by most hospitals in China, and favorable survival outcomes have been demonstrated for this protocol. However, it remains unclear whether all the high-risk areas are invaded in each NPC patient. If we include all the high-risk regions and the extension expands uniformly from the GTV, normal tissues are exposed to excessively high doses of radiation. To decrease the doses to normal tissues, reduced target volumes were applied for NPC [15-17, 33]. Lin and colleagues [15, 
16] used reduced target volumes in IMRT for NPC, and their protocol provided promising local control and survival with acceptable adverse events. Sanford and colleagues [17] designed individual delineations of the CTV for 73 NPC patients treated with IMRT; they found that the 5-year local control, regional control, and OS rates were $94 \%, 99 \%$, and $84 \%$, respectively. The reduced-volume protocol designed by Billan et al. [33] decreased the volumes of PTV of primary tumor (PTV-P) by $27.6 \%$ compared to those in RTOG 0615, and the 3-year disease-free survival was $75 \%$. Given the present situation, international guidelines introduced the principle of the " $5+5 \mathrm{~mm}$ expansion" margin from the GTV (Table 4) [27]. This protocol was based on consensus from international experts with experience in the treatment of NPC. The theoretical basis was derived from the data on the microscopic extension from recurrent NPC tumors [34] and other head-neck cancers [35,36]. Unfortunately, no clinical survival outcomes using this protocol were reported.

In addition, a better understanding of prescribed doses is needed. In the era of conventional RT, the isocenter dose was often 66-70 Gy in 30 fractions. Chau et al. $[37,38]$ used a three-dimensional treatment planning system to evaluate the actual dosimetric distribution of Ho's technique for NPC; the results showed that the D95s of the GTV and PTV in an isocenter dose of $66 \mathrm{~Gy}$ are about $57 \mathrm{~Gy}$ and $45 \mathrm{~Gy}$, respectively. Kam et al. [39] indicated that Ho's technique offers a D95 of 62.5 Gy in the GTV and 52.5 Gy in the PTV for T1 NPC patients, a D95 of 63 Gy in GTV and 57.5 Gy in PTV for T2 patients, and a D95 of 65 Gy in GTV and 55 Gy in PTV for T4 cases. Our previous study demonstrated that conventional RT techniques provide 63.8 Gy for D95 in PGTVnx, 63 Gy for D95 in PTVnx, and 42.5 Gy for D95 in PTV [40]. Although the dose distributions of conventional RT techniques were unsatisfactory, the local control rate was about $80 \%$. Based on these results, the prescribed doses of IMRT were 66-70 Gy to the GTV. However, an insufficient dose to the GTV in T3-4 NPC patients treated with IMRT was related to poor local control [41]. Thus, data of appropriate prescribed doses for target volumes are lacking.

Accordingly, we divided the tumor and the anatomic sites around the tumor into three or four risk target volumes based on the orderly stepwise pattern of tumor spread (Table 1). High-risk PGTVnx and PGTVnd were irradiated with high doses, intermediate-risk PTVnx and PTVna were irradiated with intermediate doses, and low-risk PTV was irradiated with low doses. If the RLNs were large or resistance to IC, we add PGTVrpn to PGTVnx and administered a higher dose. Using our protocol, the high dose was more concentrated in the GTV, and the areas receiving a low dose were decreased. The BEDs of our protocol were similar to those of the IMRT dose regimens in the China and 0615 protocols (Table 4). Moreover, the corresponding EQDs2 of our protocol were $73.44,63.53,60$, and $49.73 \mathrm{~Gy}$ in $70.5 \mathrm{~Gy} / 30 \mathrm{~F}$, 63Gy/30F, 60Gy/30F and 51Gy/30F, respectively. Notably, the LRRFS, DMFS, PFS, and OS rates at 5 years were $92.2 \%, 92.1 \%, 85.9 \%$, and $86.3 \%$, respectively.

Although the present protocol provided favorable dosimetric parameters and survival outcomes with fewer acute and late adverse events, several limitations of our study should be noted. First, as this was a single-center study, all the limitations associated with single centers apply. Second, due to the retrospective nature of the study, it provided relatively low power to indicate the superiority of our protocol. Third, acute adverse events were assessed according to medical records; no quality-of-life assessments were performed for long-term survivors. Moreover, the effect of IC has not been considered in our protocol. Thus, our protocol should be regarded as preliminary. Our revised protocol should be verified in future prospective and large-sample clinical trials.

\section{Conclusion}

We presented a protocol of different risk target volumes and corresponding prescribed doses for IMRT in NPC patients. Our protocol resulted in superior dose distributions for reducing high-dose volumes in the GTV and decreasing low-dose volumes in normal tissues. The present regimen provided favorable long-term survival outcomes with acceptable acute and late adverse events. Therefore, this protocol can be recommended for the treatment of NPC.

\section{Funding}

This study was supported by grants from the Medical and Health Science and Technology Program of Zhejiang Province (No. 2020KY084, No. 2019KY041, No. 2013KYB033, No. 2009B026, No. 2006A016, No. 2005B012, and No. 2004B014) and the National Natural Science Foundation of China (No. 81502647).

\section{Abbreviations}

AC: adjuvant chemotherapy; BED: biological effective dose; CT: computed tomography; CTV: clinical target volume; CTVna: CTV of neck area; CTVnx: CTV of nasopharynx; DMFS: distant metastasis-free survival; DVH: dose-volume histogram; EQDs2: equivalent doses in 2 Gy/fraction; GTV: gross tumor volume; GTVnd: GTV of cervical 
lymph nodes; GTVnx: GTV of nasopharynx; GTVrpn: GTV of retropharyngeal lymph nodes; IC: induction chemotherapy; IMRT: intensity-modulated radiotherapy; LRRFS: locoregional relapse-free survival; MRI: magnetic resonance imaging; NPC: nasopharyngeal cancer; OAR: organ at risk; OS: overall survival; PFS: progression-free survival; PGTVnd: planning target volume of GTV in cervical lymph nodes; PGTVnx: planning target volume of GTV in nasopharynx; PGTVrpn: planning target volume of GTV in retropharyngeal lymph nodes; PTV: planning target volume; PTVna: PTV of neck area; PTVnx: PTV of nasopharynx; RLN: retropharyngeal lymph node; RT: radiotherapy; SEER: Surveillance, Epidemiology, and End Results; SEQ: sequential; SIB: simultaneously integrated boost; V30: the volume received 30; V40: the volume received $40 \mathrm{~Gy}$.

\section{Competing Interests}

The authors have declared that no competing interest exists.

\section{References}

1. Tang LL, Chen WQ, Xue WQ, et al. Global trends in incidence and mortality of nasopharyngeal carcinoma. Cancer Lett. 2016; 374: 22-30.

2. Bray F, Ferlay J, Soerjomataram I, et al. Global cancer statistics 2018: GLOBOCAN estimates of incidence and mortality worldwide for 36 cancers in 185 countries. CA Cancer J Clin. 2018; 68: 394-424.

3. Lee AW, Foo W, Law SC, et al. Staging of nasopharyngeal carcinoma: from Ho's to the new UICC system. Int J Cancer. 1999; 84: 179-87.

4. Al-Sarraf M, Reddy MS. Nasopharyngeal carcinoma. Curr Treat Options Oncol. 2002; 3: 21-32.

5. Chua DT, Ma J, Sham JS, et al. Improvement of survival after addition of induction chemotherapy to radiotherapy in patients with early-stage nasopharyngeal carcinoma: subgroup analysis of two Phase III trials. Int J Radiat Oncol Biol Phys. 2006; 65: 1300-6.

6. Lee AW, Sze WM, Au JS, et al. Treatment results for nasopharyngeal carcinoma in the modern era: the Hong Kong experience. Int J Radiat Oncol Biol Phys. 2005; 61: 1107-16.

7. YI JL, Gao L, Huang XD, et al. Nasopharyngeal carcinoma treated by radical radiotherapy alone: ten-year experience of a single institution. Int J Radiat Oncol Biol Phys. 2006; 65: 161-8.

8. Liao W, Zhou HH, Fan SN, et al. Comparison of significant carotid stenosis for nasopharyngeal carcinoma between intensity-modulated radiotherapy and conventional two-dimensional radiotherapy. Sci Rep. 2018; 8: 13899.

9. Zhao Z, Lan Y, Bai S, et al. Late-onset radiation-induced optic neuropathy after radiotherapy for nasopharyngeal carcinoma. J Clin Neurosci. 2013; 20: 702-6.

10. Fang $\mathrm{W}, \mathrm{Gu} \mathrm{B}$, Jing $\mathrm{X}$, et al. Late-onset cystic brain necrosis after radiotherapy for nasopharyngeal carcinoma. Jpn J Clin Oncol. 2017; 47: 499-504.

11. Kam MK, Chau RM, Suen J, et al. Intensity-modulated radiotherapy in nasopharyngeal carcinoma: dosimetric advantage over conventional plans and feasibility of dose escalation. Int J Radiat Oncol Biol Phys. 2003; 56: 145-57.

12. Fangzheng $W$, Chuner J, Haiyan $Q$, et al. Survival without concurrent chemotherapy for locoregionally advanced nasopharyngeal carcinoma treated with induction chemotherapy plus intensity-modulated radiotherapy: single-center experience from an endemic area. Medicine (Baltimore). 2019; 98: e18484.

13. Wang $\mathrm{R}, \mathrm{Wu} \mathrm{F}, \mathrm{Lu} \mathrm{H}$, et al. Definitive intensity modulated radiation therapy for nasopharyngeal carcinoma: long-term outcome of a multi-center prospective study. J Cancer Res Clin Oncol. 2013;139: 139-45.
14. Lin L, Yao JJ, Zhou GQ, et al. The efficacy and toxicity of individualized intensity modulated radiation therapy based on the tumor extension patterns of nasopharyngeal carcinoma. Oncotarget. 2016; 7: 20680-90.

15. Lin S, Pan J, Han L, et al. Nasopharyngeal carcinoma treated with reduced-volume intensity-modulated radiation therapy: report on the 3-year outcome of a prospective series. Int J Radiat Oncol Biol Phys. 2009; 75: $1071-8$.

16. Lin S, Pan J, Han L, et al. Update report of nasopharyngeal carcinoma treated with reduced-volume intensity-modulated radiation therapy and hypothesis of the optimal margin. Radiother Oncol. 2014; 110: 385-9.

17. Sanford NN, Lau J, Lam MB, et al. Individualization of clinical target volume delineation based on stepwise spread of nasopharyngeal carcinoma: outcome of more than a decade of clinical experience. Int J Radiat Oncol Biol Phys. 2019; 103: 654-68.

18. Wang F, Jiang C, Wang L, et al. Influence of concurrent chemotherapy on locoregionally advanced nasopharyngeal carcinoma treated with neoadjuvant chemotherapy plus intensity-modulated radiotherapy: a retrospective matched analysis. Sci Rep. 2020; 10: 2489.

19. Beadle BM, Liao KP, Elting LS, et al. Improved survival using intensity-modulated radiation therapy in head and neck cancer: a SEER-Medicare analysis. Cancer. 2014; 120: 702-10.

20. Chen SW, Yang SN, Liang JA, et al. Comparative dosimetric study of two strategies of intensity-modulated radiotherapy in nasopharyngeal cancer. Med Dosim. 2005; 30: 219-27.

21. Songthong AP, Kannarunimit D, Chakkabat C, et al. A randomized phase II/III study of adverse events between sequential (SEQ) versus simultaneous integrated boost (SIB) intensity modulated radiation therapy (IMRT) in nasopharyngeal: preliminary result on acute adverse events. Radiat Oncol. 2015; 10: 166.

22. Lertbutsayanukul C, Prayongrat A, Kannarunimit D, et al. A randomized phase III study between sequential versus simultaneous integrated boost intensity-modulated radiation therapy in nasopharyngeal carcinoma. Strahlenther Onkol. 2018; 194: 375-85.

23. Chung JB, Lee JW, Kim JS, et al. Comparison of target coverage and dose to organ at risk between simultaneous integrated-boost whole-field intensity-modulated radiation therapy and junctioned intensity-modulated radiation therapy with a conventional radiotherapy field in treatment of nasopharyngeal carcinoma. Radiol Phys Technol. 2011; 4: 180-4.

24. Tian YM, Liu MZ, Zeng L, et al. Long-term outcome and pattern of failure for patients with nasopharyngeal carcinoma treated with intensity-modulated radiotherapy. Head Neck. 2019; 41: 1246-52.

25. Zhu W, Chen F, Li J, et al. Dosimetric parameters associated with conductive or sensorineural hearing loss 5 years after intensity-modulated radiation therapy in nasopharyngeal carcinoma. Acta Otolaryngol. 2019; 139: 263-8.

26. Ng WT, Lee MC, Chang AT, et al. The impact of dosimetric inadequacy on treatment outcome of nasopharyngeal carcinoma with IMRT. Oral Oncol. 2014; 50: 506-12.

27. Lee $\mathrm{AW}, \mathrm{Ng}$ WT, Pan JJ, et al. International guideline for the delineation of the clinical target volumes (CTV) for nasopharyngeal carcinoma. Radiother Oncol. 2018; 126: 25-36.

28. [Internet] China Nasopharyngeal Carcinoma Clinical Staging Working Committee. Expert consensus on the target area and dose design guidelines for intensity modulated radiotherapy of nasopharyngeal carcinoma 2010. Chin J Radiat Oncol 2011; 20: 267-69 [in Chinese]. http:// zhfszlxzz.yiigle.com/CN11303020112004/424433.htm.

29. Lee N, Harris J, Garden AS, et al. Intensity-modulated radiation therapy with or without chemotherapy for nasopharyngeal carcinoma: Radiation Therapy Oncology Group Phase II trial 0225. J Clin Oncol. 2009; 27: 368490.

30. Dubrulle F, Souillard R, Hermans R. Extension patterns of nasopharyngeal carcinoma. Eur Radiol. 2007; 17: 2622-30.

31. Liang SB, Sun Y, Liu LZ, et al. Extension of local disease in nasopharyngeal carcinoma detected by magnetic resonance imaging: improvement of clinical target volume delineation. Int J Radiat Oncol Biol Phys. 2009; 75: 742-50.

32. Li WF, Sun $Y$, Chem $M$, et al. Locoregional extension patterns of nasopharyngeal carcinoma and suggestions for clinical target volume delineation. Clin J Cancer. 2012; 31: 579-87.

33. Billan S, Bakouche V, Borzov E, et al. Long-term outcomes in patients with nasopharyngeal carcinoma treated with reduced-volume conformal radiotherapy: a retrospective cohort study. J Med Imaging Radiat Oncol. 2018; 62: 562-7.

34. Chan JY, Wong ST, Wei WI. Whole-organ histopathological study of recurrent nasopharyngeal carcinoma. Laryngoscope. 2014; 124: 446-50.

35. Fleury B, Thariat J, Barnoud R, et al. Microscopic extensions of head and neck squamous cell carcinoma: impact for clinical target volume definition. Cancer Radiother. 2014; 18: 666-71 [in French]. 
36. Ligtenberg H, Jager EA, Caldas-Magalhaes J, et al. Modality-specific target definition for laryngeal and hypopharyngeal cancer on FDG-PET, CT and MRI. Radiother Oncol. 2017; 123: 63-70.

37. Chau, RM, Teo PM, Choi PH, et al. Three-dimensional dosimetric evaluation of a conventional radiotherapy technique for treatment of nasopharyngeal carcinoma. Radiother Oncol. 2001; 58: 143-53.

38. Chau RM, Teo PM, Kam MK, et al. Dosimetric comparison between 2-dimensional radiation therapy and intensity-modulated radiation therapy in treatment of advanced T-stage nasopharyngeal carcinoma: to treat less or more in the planning organ-at-risk volume of the brainstem and spinal cord. Med Dosim. 2007; 32: 263-70.

39. Kam, MK, Chau RM, Suen J, et al. Intensity-modulated radiotherapy in nasopharyngeal carcinoma: dosimetric advantage over conventional plans and feasibility of dose escalation. Int J Radiat Oncol Biol Phys. 2003; 56:145-57.

40. Fangzheng W, Chuner J, Lei W, et al. Outcome and long-term efficacy of four facio-cervical fields conformal radiotherapy for nasopharyngeal carcinoma. Oncotarget. 2017; 8: 39756-65.

41. Xu F, Hu C, He X. Impact of minimum point dose on local control and toxicity in T3-4 nasopharyngeal carcinoma treated with intensity-modulated radiation therapy plus chemotherapy. Jpn J Clin Oncol. 2018; 48: 265-71. 